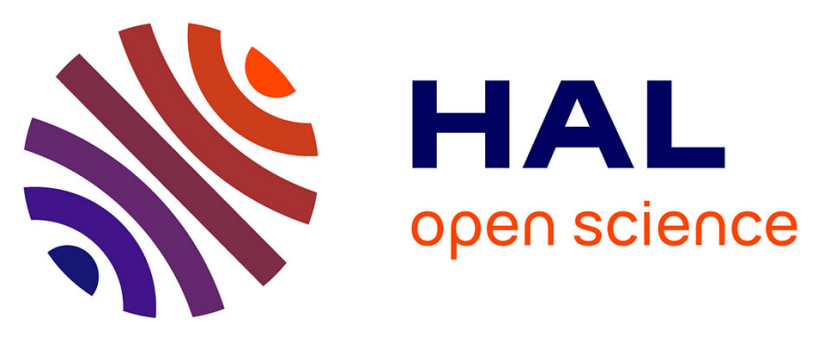

\title{
Liquid-Gated Transistors Based on Reduced Graphene Oxide for Flexible and Wearable Electronics
}

\author{
Rafael Furlan de Oliveira, Pietro Antonio Livio, Veronica Montes-garcía, \\ Stefano Ippolito, Matilde Eredia, Pablo Fanjul-bolado, María Begoña \\ González García, Stefano Casalini, Paolo Samorì
}

\section{To cite this version:}

Rafael Furlan de Oliveira, Pietro Antonio Livio, Veronica Montes-garcía, Stefano Ippolito, Matilde Eredia, et al.. Liquid-Gated Transistors Based on Reduced Graphene Oxide for Flexible and Wearable Electronics. Advanced Functional Materials, 2019, 29 (46), pp.1905375. 10.1002/adfm.201905375. hal-02362516

\section{HAL Id: hal-02362516 https://hal.science/hal-02362516}

Submitted on 13 Nov 2019

HAL is a multi-disciplinary open access archive for the deposit and dissemination of scientific research documents, whether they are published or not. The documents may come from teaching and research institutions in France or abroad, or from public or private research centers.
L'archive ouverte pluridisciplinaire HAL, est destinée au dépôt et à la diffusion de documents scientifiques de niveau recherche, publiés ou non, émanant des établissements d'enseignement et de recherche français ou étrangers, des laboratoires publics ou privés. 


\section{WILEY-VCH}

Liquid-gated transistors based on reduced graphene oxide for flexible and wearable electronics

Rafael Furlan de Oliveira, Pietro Antonio Livio, Verónica Montes-García, Stefano Ippolito, Matilde Eredia, Pablo Fanjul-Bolado, María Begoña González García, Stefano Casalini*, Paolo Samori*

Dr. R.F. Oliveira, P.A. Livio, Dr. V. Montes-García, S. Ippolito, Dr. M. Eredia, Dr. S. Casalini, Prof. Dr. P. Samorì

Université de Strasbourg, CNRS, ISIS UMR 7006, 8 allée Gaspard Monge, F-67000

Strasbourg, France.

E-mail: casalini@unistra.fr, samorì,unistra.fr

Dr. P. Fanjul-Bolado, Dr. M.B. González García

Metrohm DropSens,S.L., Ed.CEEI, Parque Tecnológico de Asturias, 33428-Llanera, Asturias, Spain.

Keywords: Reduced Graphene Oxide, Liquid-Gated Transistors, Flexible Electronics, Wearable Electronics, Lateral flow 


\section{WILEY-VCH}

\section{Abstract}

Graphene is regarded as the ultimate material for future flexible, high-performance and wearable electronics. Herein, we report a novel, robust, all-green, highly reliable (yield $\geq$ 99\%) and up-scalable technology for wearable applications comprising reduced graphene oxide (rGO) thin-films as electroactive component in liquid-gated transistors (LGTs). Although the intrinsic electrical performance of rGO cannot compete with CVD graphene, its ease processability, excellent surface reactivity, and large-area coverage make rGO a formidable material for future flexible and wearable applications. We have established a novel protocol towards the high-yield fabrication of flexible rGO LGTs combining high robustness $(>1.5 \mathrm{~h}$ of continuous operation) with state-of-the-art performances, being similar to those of their rigid counterparts operated under liquid gating, including field-effect mobility of ca. 10${ }^{1} \mathrm{~cm}^{2} \mathrm{~V}^{-1} \mathrm{~s}^{-1}$ and transconductance of ca. $25 \mu \mathrm{S}$. Permeable membranes have been proved crucial to operate flexible LGTs under mechanical stress and with reduced amounts of solution $(<20 \mu \mathrm{L})$. Our rGO LGTs were operated in artificial sweat exploiting two different layouts based on lateral-flow paper fluidics. These approaches pave the road towards future real-time tracking of perspiration via a simple and cost-effective approach. The reported findings contribute to the robust and scalable production of novel graphene-based flexible devices, whose features fulfill the requirements of wearable electronics. 


\section{WILEY-VCH}

\section{Introduction}

The pioneering work on graphene by Geim and Novoselov ${ }^{[1]}$ have stimulated an intense research endeavor on graphene-related materials (GRMs) with the aim of gaining an in-depth insight into their outstanding electrical/electronic, mechanical, optical and thermal properties for applications in (opto)electronics, energy, sensing and composites ${ }^{[2]}$. GRMs are envisioned to improve the existing characteristics and performance of products and applications already in the market, and also to enable the emergence of novel technologies and functionalities. In electronics, one of the expected graphene-driven breakthroughs consists in the development of flexible, transparent and wearable devices ${ }^{[3,4]}$. Several applications targeting the use of graphene or GRMs for the realization of wearables devices have already been demonstrated. For example, Lu, Akinwande and co-workers reported a graphene e-tattoo capable to track the skin hydration and the body temperature ${ }^{[5]}$. Lee et al. demonstrated the efficient detection of $\mathrm{pH}$, glucose, and temperature on the skin of diabetic individuals, which can benefit from the automatic transcutaneous delivery of Metformin if demanded ${ }^{[6]}$. Furthermore, graphene elenses have been developed to track glucose concentration and the ocular pressure, independently ${ }^{[7]}$. Such significant progress towards wearable applications has been achieved by using chemical vapor deposited (CVD) graphene, which guarantees top-class device performances yet combined with some technological limitations ${ }^{[3]}$. Reduced graphene oxide (rGO) is an alternative electroactive material. Despite it cannot compete with CVD graphene in terms of electrical properties, its easy processability, rich surface functionality, and largearea coverage render $\mathrm{rGO}$ an interesting compromise ${ }^{[8,9]}$.

The production of rGO thin-films from graphene oxide (GO) for wearable and flexible devices involves two critical aspects, i) the GO casting technology, and ii) its reduction. Many approaches have been exploited to assemble GO in thin films such as drop-casting ${ }^{[10,11]}$, dipcoating $^{[12]}$, rapid freezing spray coating ${ }^{[12]}$ and vacuum filtration. These methods can allow to process GO onto large-area, flexible and irregular substrates ${ }^{[13]}$. Many of these protocols show 


\section{WILEY-VCH}

technologically appealing characteristics, such as low-cost and batch processing of GO thinfilms, which are key for industrial production of related devices. In order to reduce GO, chemical, thermal, electrical and electrochemical approaches have been employed, each of them possessing pros and cons depending on the envisioned application ${ }^{[14]}$. The rich surface functionality represents one among the most important characteristics of rGO, which makes it particularly suitable for applications in (bio-)sensing. The sensing efficiency of rGO-based devices can be finely tuned by covalently tethering receptors of specific analytes ${ }^{[15]}$ enabling the selective interaction with $\mathrm{DNA}^{[16]}$, odorant binding protein ${ }^{[17]}$, urea, metal cations ${ }^{[18]}\left(\mathrm{Cu}^{2+}\right.$, $\left.\mathrm{Mg}^{2+}, \mathrm{Ca}^{2+}, \mathrm{Hg}^{2+}, \mathrm{Cd}^{2+}\right)^{[19]}, \mathrm{pH}$, bovine serum albumin ${ }^{[20]}$ and acetylcholine ${ }^{[21]}$. Zhang et al. have recently demonstrated an alternative approach capable to merge large-area, flexible field-effect transistors and the sensing features of functionalized $\mathrm{rGO}^{[22]}$. This approach has been challenged for the (bio-)chemical detection of catecholamines (viz. dopamine, epinephrine, etc.) produced during the depolarization of PC12 cells. The reported strategy was further improved to achieve an all-rGO device capable to detect avidin in benchmark solutions by using a biotinylated membrane placed on top of the rGO thin-film ${ }^{[23]}$. The combination of chemical reactivity, easy processability and satisfactory electrical properties makes rGO an ideal component for the development of wearable sensors and biosensors. However, towards the emergence of a real "wearable technology", it is mandatory to devise efficient and scalable protocols for the fabrication of flexible graphene-based devices that can be operated in liquid, especially in sweat. The human sweat represents a rich source of physiological information for health monitoring, with a few examples of graphene devices employed for this purpose ${ }^{[14]}$.

Here, we develop flexible rGO liquid-gated transistors (LGTs) capable to operate in sweat by taking full advantage of flexible LGTs for sensing, such as its intrinsic high signal amplification capability and ability to operate at low voltages ${ }^{[24-26]}$. Towards this end, a fully green (aqueous-based), highly reliable (yield $\geq 99 \%$ ) industrial-compatible device fabrication 


\section{WILEY-VCH}

strategy has been devised which combines different established device nano- and microfabrication approaches including the laser-ablation patterning of flexible electrodes, implementation of LGTs comprising a coplanar gate_(CoG) electrode, in-situ electrochemical reduction of GO cast over large areas, as well as confinement and transport of solutions to the device by means of permeable membranes.

We provide evidence for the robustness of our flexible rGO LGTs $(>1.5 \mathrm{~h}$ of continuous operation), under severe mechanical stress and utilizing small amounts of solution $(<20 \mu \mathrm{L})$. Finally, two simple device layouts based on lateral-flow paper fluidics for solution transportation have been demonstrated for the real-time monitoring of sweat by means of rGO LGTs.

\section{Results and discussion}

The fabrication protocol of standard, top-gated liquid-gated transistors (TG-LGTs) based on rGO comprises 4 steps (Fig. 1a, Fig. S1a,c). This approach relies on the use of 3(aminopropyl)triethoxysilane (APTES) as the adhesive primer to improve the otherwise modest adhesion of $\mathrm{GO}$ onto $\mathrm{SiO}_{2}$. By enriching the $\mathrm{SiO}_{2}$ surface with positively charged amino groups, the GO grafting onto the substrate of interest is fostered by electrostatic interactions ${ }^{[27,28]}$. Moreover, among the wide portfolio of approaches which have been previously demonstrated to reduce $\mathrm{GO}^{[14]}$, we opted to use the electrochemical one because of its intrinsic advantages for flexible and wearable devices ${ }^{[12,29-33]}$, since it allows in-situ fast reaction with a high spatial resolution by using friendly chemicals and inexpensive set-ups. Although this approach has been known since many years ${ }^{[29]}$, its exploitation has been mostly limited to electrochemical applications rather than for devices like transitors ${ }^{[34-38]}$. Our GO electrochemical reduction has been carried out in bi-distilled water as illustrated in Fig. 1 b. Detailed information about the GO electrochemical reduction can be found in the Experimental Section and in the SI (Fig. S2). The GO reduction into rGO is accompanied by a 


\section{WILEY-VCH}

dramatic drop in electrical resistivity $(\varrho)$, namely from $0.1 \mathrm{M} \Omega \cdot \mathrm{cm}$ to $0.7-1.0 \Omega \cdot \mathrm{cm}$. These values are compatible with other GO reduction methods and electrochemical strategies ${ }^{[37,39,40]}$. The optical microscopy image in Fig. 1c reveals that the GO electrochemical reduction yields a spatial resolution of $c a .50-100 \mu \mathrm{m}$ around the interdigitated electrodes (IDEs), keeping the residual areas of the chip electrically insulated. This is advantageous to avoid possible device cross-talk in a LGT array chip. The spatial resolution of such in-situ, large-area, electrochemical rGO patterning can be tuned with the potential sweeping rate.

SEM images of rGO thin-films in Fig. 1d (see inset for details) revealed the absence of macro-scale defects on the surface, such as holes or wrinkles, commonly observed when harsh reductive processes are employed ${ }^{[14,30]}$. At the mesoscale, AFM images displayed the typical multilayer-flake morphology of $\mathrm{rGO}^{[10]}$, with thickness of $c a .2-3 \mathrm{~nm}$ and root-mean square roughness $\left(R_{r m s}\right)$ of $0.15 \pm 0.02 \mathrm{~nm}$ over a $5 \mu \mathrm{m} \times 5 \mu \mathrm{m}$ (Fig. S3 and Fig. 1e, respectively). The rGO grade of homogeneity upon the test pattern was evaluated by means of Raman spectroscopy (Fig. 1f) and XPS (Fig. S4). Raman mapping (Fig. 1f, inset) of the D/G band intensity ratio spans from 1.01 (namely pure GO) to 1.52 (namely pure rGO), in agreement with the literature ${ }^{[41]}$. The Raman mapping indicates a clear distinction between the GO and rGO regions over the chip, with the accentuated presence of rGO within the IDE channel. Alongside, unambiguous evidence of the absence of film discontinuity, especially at the IDE edges responsible for electrical charge injection/withdrawal, has been observed. Surface Raman mapping also corroborated the above-mentioned GO film thickness extracted from AFM providing information of the film homogeneity over large areas (see Fig. S5). XPS data revealed a substantial decrease in the $\mathrm{C}-\mathrm{O}$ content after the $\mathrm{GO}$ electrochemical reduction (Fig. S4). In addition to the general spectroscopic and morphological characterizations, the electrical characteristics of the coated test patterns have been assessed by fabricating twoterminal devices and testing them when operating in either dry state or in bi-distilled water. Fig. $1 \mathrm{~g}$ shows a clear ohmic response while sweeping the IDE bias within $-0.1 \mathrm{~V}$ to $0.1 \mathrm{~V}$. 


\section{WILEY-VCH}

The calculated $\varrho$ corresponds to $1.8 \Omega \cdot \mathrm{cm}$ and $0.8 \Omega \cdot \mathrm{cm}$ in dry and wet state, respectively.

Such evidence demonstrates that rGO can be operated in water without any parasitic current due to liquid environment ${ }^{[42,43]}$. Therefore, $\mathrm{rGO}$ thin-films could be successfully employed as active material in TG-LGTs on $\mathrm{Si} / \mathrm{SiO}_{2}$ (Fig. S6) as well as on glass substrates (Fig. S7). Although the development of glass-based rGO LGTs are beyond the scope of this manuscript, such devices are advantageous for future bio- and/or opto-electronic applications, where transparency in the visible range is required.

The application of LGTs for wearable electronics demands technological solutions to some traditional features exhibited by standard devices, such as their top gate (TG) architecture, the use of rigid substrates, and a polydimethylsiloxane (PDMS) reservoir for solution confinement (Fig. S6). To tackle these limitations, we have adopted a fabrication protocol based on a co-planar gate $(\mathrm{CoG})$ architecture onto $\mathrm{Si} / \mathrm{SiO}_{2}$ for direct comparison with the standard TG-LGTs. Once validated, we have revised our fabrication method by supporting the device onto polyethylene terephthalate (PET) substrates. The CoG represents a compact architecture that can be easily connected to portable circuitry. The CoG-LGT design consists of a squared gate electrode $\left(0.09 \mathrm{~cm}^{2}\right.$ area) placed $4 \mathrm{~mm}$ distant from the IDEs (Fig. S8 for details). Two approaches for the GO deposition have been successfully exploited: i) the PDMS-assisted micro-contact printing, ${ }^{[27,44]}$ and ii) substrate partial dipping. Both approaches succeeded by using APTES aqueous solution instead of organic solvents (e.g. toluene). The former strategy consists of stamping APTES by means of a PDMS mold directly onto the IDEs area (Fig. S9), whereas the latter requires the IDEs immersion into the APTES solution (Fig. 2a). Although both processes are green and well-suited for GO patterning, the partial dipping method exhibited higher reproducibility than the micro-contact printing one.

For the sake of comparison, we recorded I-V transfer (Fig. 2b,c) and output characteristics (Fig. 2d,e) in bi-distilled for the same device in TG and CoG architectures. A PDMS reservoir was utilized to confine the solution on top of the device. The replacement of PDMS reservoir 


\section{WILEY-VCH}

by a permeable membrane for the device is presented hereafter. For TG operation an Au wire was employed as the gate electrode. I-V transfer characteristics show the usual ambipolar behavior, whose the minimum source-drain current $\left(I_{D S}^{\min }\right)$ is close to $0.5 \mathrm{~V}$ in both architectures. A small $I_{D S}$ rise occurs between $-0.4 \mathrm{~V}$ and $-0.35 \mathrm{~V}$ and it is related of the slow polarization process of the bi-distilled at the two main LGT interfaces (i.e. gate-electrolyte and rGO-electrolyte). The $I_{D S}$ modulation is well-defined for both architectures with a slight improvement for the $\mathrm{CoG}$ mode. As a result, the hole field-effect mobility $\left(\mu_{h}\right)$ was found to amount to $0.38( \pm 0.08) \mathrm{cm}^{2} \mathrm{~V}^{-1} \mathrm{~s}^{-1}$ and $1.7( \pm 0.5) \mathrm{cm}^{2} \mathrm{~V}^{-1} \mathrm{~s}^{-1}$ for TG-LGT and CoG-LGT, respectively, whereas the electron mobility $\left(\mu_{e}\right)$ resulted similar in both architectures $\left(\mu_{e}=0.17 \mathrm{~cm}^{2} \mathrm{~V}^{-1} \mathrm{~s}^{-1}\right.$ and $\mu_{e}=0.13 \mathrm{~cm}^{2} \mathrm{~V}^{-1} \mathrm{~s}^{-1}$, respectively $)$. Coherently, the $I_{D S}^{\max } / I_{D S}^{\min }$ ratio is slightly higher for the CoG-LGT than for TG-LGT (i.e. 3.1 versus 2.4). Details on the calculation of the charge carrier mobility are shown in the SI. The I-V output characteristics have not presented any substantial discrepancies among the two LGT architectures. In fact, no $I_{D S}$ saturation was recorded, and no significant contact resistance issues have been observed. The leakage current $\left(I_{G S}\right)$ of such devices exhibited no indications of electrochemical processes (viz. redox peaks) attesting for material degradation, being also almost two orders of magnitude lower than $I_{D S}$ (Fig. S10). Our results demonstrate that the standard LGT technology can be efficiently transferred to a more integrated CoG-based architecture without jeopardizing the device performance.

When moving from rigid $\left(\mathrm{Si} / \mathrm{SiO}_{2}\right)$ to flexible (PET) substrates, special attention was paid to the electrode fabrication and the GO coating. Here, flexible PET test patterns were produced by laser ablation aiming to low-cost and batch electrode prototyping. Although other efficient methods for electrode patterning are known, our protocol can allow a straightforward industrial scale-up. Significantly, our approach guarantees a similar spatial IDE/channel resolution with respect to standard photolithography possessing a high-yield (>99\%) 


\section{WILEY-VCH}

fabrication compliant with plastic substrates. The laser ablation of PET substrates, however, yields a rougher surface ( $\sigma_{r m s}>60 \mathrm{~nm}$, see Fig. $\left.\mathrm{S} 11\right)$ compared to $\mathrm{Si} / \mathrm{SiO}_{2}$, which can likely affect the uniformity of the deposited films and consequently the final device performance ${ }^{[45]}$. For this reason, the standard APTES adhesive primer for GO patterning has been replaced by a polycation, namely poly(diallylmethylammonium chloride) (PDDA). This is advantageous because PDDA is water soluble and it enriches the substrate surface with a higher amount of amino groups compared to APTES ${ }^{[46]}$, without requiring previous UV/ozone surface activation. The GO deposition and reduction were accomplished by following the previously described steps for the CoG-LGT fabrication (Fig. 3a). Alongside the standard check of electrical resistivity, optical microscopy imaging in dark field provides also evidence for the effective GO coating and reduction onto PET (Fig. 3b).

The I-V transfer and output characteristics for PET/PDDA/rGO CoG-LGTs have been acquired (Fig. 3c and Fig. S12, respectively). As shown on devices supported onto $\mathrm{Si} / \mathrm{SiO}_{2}$ substrates, the CoG architecture ensures efficient channel electrical modulation featuring $\mu_{h}$ and $\mu_{e}$ of $0.26( \pm 0.02) \mathrm{cm}^{2} \mathrm{~V}^{-1} \mathrm{~s}^{-1}$ and $0.027( \pm 0.005) \mathrm{cm}^{2} \mathrm{~V}^{-1} \mathrm{~s}^{-1}$, respectively, and an $I_{D S}^{\max } / I_{D S}^{\min }$ ratio of $c a .4$.

Aiming to gain greater insights into the electrical characteristics of rGO LGTs, especially about the liquid/rGO interface charge dynamics, we have performed electrochemical impedance spectroscopy (EIS) and pulsed DC stimulation studies. Figure 3d portrays the EIS phase spectrum and the corresponding capacitance curve for a device biased on the CoG with $-0.3 \mathrm{~V}$. The constant, capacitive-like $\left(\mathrm{ca} .-75^{\circ}\right)$ impedance phase at low frequencies $(<10 \mathrm{~Hz})$ indicates the formation of electrical double-layers at the LGT interfaces ${ }^{[4]}$. The corresponding measured capacitance amounts to $5 \mu \mathrm{F} / \mathrm{cm}^{2}$ at the phase plateau (Fig. 3d), being in good agreement with other LGTs ${ }^{[20]}$. The continuous capacitance increase at low frequencies at a fixed gate bias suggests the ion incorporation into the rGO film layer structure which 


\section{WILEY-VCH}

contributes to such elevated capacitances ${ }^{[47-49]}$. This is an expected characteristic considering recent reports on ionic confinement in $\mathrm{rGO}$ films ${ }^{[50]}$. Another important feature is the so-called potentiometric sensitivity that expresses the capability of the device to sense voltage fluctuations ${ }^{[51,52]}$. Within this context, a series of positive and negative $\Delta V_{G S}$ varying from $200 \mathrm{mV}$ to $2 \mathrm{mV}$ has been applied while recording the device $I_{D S}$ as a function of time (Fig. 3e). This electrical test has been carried out by fixing the initial source-gate voltage $\left(V_{G S}\right)$ at $-0.2 \mathrm{~V}$, which guarantees the LGT highest transconductance $\left(I_{D S} / V_{G S}=g_{m}=25 \mu \mathrm{S}\right)$. The device showed a clear sensitivity down to $2 \mathrm{mV}$, featuring a response with a signal-to-noise ratio $(\mathrm{S} / \mathrm{N}) \simeq 31$. Here, rGO LGTs responded to pulsed potential stimulation in a time-scale of $c a .2 \mathrm{~s}$. Such an ability to sense small $V_{G S}$ variations down to a few $\mathrm{mV}$ with fast response is of great value for the development of potentiometric sensors and biosensors in liquid medium $^{[53,54]}$. The device potentiometric response is fully operational also at positive $V_{G S}$, as illustrated in Fig. S13. Finally, PET-based rGO CoG-LGT has shown an impressive electrical robustness withstanding $c a .800$ consecutive transfer scans ( $>1.5$ h operation) without presenting failures. Although the presence of bias stress was monitored during operation, the device remained functioning with $g_{m}$ reduction (at $-0.3 \mathrm{~V}$ ) of only $25 \%$ (Fig. S14).

In wearable electronics, especially for health care monitoring, the human sweat is regarded as as a rich source of physiological information ${ }^{[55-58]}$. Several devices have been developed to monitor different metabolites in sweat. Among them, different smart e-platforms rely on electrical and electrochemical transducers operating under very specific conditions ${ }^{[59-61]}$. Here, to test the technological potential of rGO LGTs for wearable applications, we have evaluated the device operability in artificial sweat. The artificial sweat mimics the human sweat in terms of its chemical properties (viz. ionic strength and $\mathrm{pH}$ ). Firstly, the LGT operational potentials have been limited to the range of $\pm 400 \mathrm{mV}$, since higher potentials have been found to damage the device (data not shown). Aiming at wearables based on LGTs, an additional strategy consists in the replacement of their traditional PDMS reservoir by a sweat-permeable 


\section{WILEY-VCH}

membrane. As illustrated in Fig. 4a, a permeable membrane, either polymeric or paper-based, is able to confine a liquid drop onto the device during operation. The use of permeable membranes also enables the use of smaller volumes of solution (down to 10-20 $\mu \mathrm{L}$ ) to operate LGTs with respect to standard PDMS reservoirs ( $\geq 100 \mu \mathrm{L}$ ). This method represents a smart strategy to electrically bridge source-drain and gate electrodes in CoG-LGTs aiming to meet the stringent requirements of wearable technology.

The operational characteristics of rGO-based CoG-LGTs on PET by using a permeable membrane have been extensively investigated. Firstly, the dynamics of the device wetting has been evaluated by recording time-dependent $I_{D S}$ and $I_{G S}$ upon sweat addition. From Fig. 4b, a small addition of sweat (down to $20 \mu \mathrm{L}$ ) on the membrane causes $I_{D S}$ and $I_{G S}$ spikes, followed by a steady-state current. Such a transient response occurs on the time-scale of a few seconds $(<5 \mathrm{~s})$ for the tested membranes (Fig. S15). No significant current fluctuations have been recorded. The reported devices have also demonstrated a stable response during transfer scans (Fig. 4c and Fig. S16). As previously observed for TG-LGTs (Fig. S6), the device operation in solutions with higher ionic strength shifts the $I_{D S}^{\min }$ value towards $0 \mathrm{~V}$. This shift stems from the abrupt change of ionic strength in the liquid when moving from bi-distilled water to $\mathrm{KCl}$ $0.1 \mathrm{M}$ and/or sweat, because it decreases the well-known Debye length (i.e. length of the electrostatic screening of a charged particle in solution). This phenomenon is responsible for the more efficient screening of dopants present into or close to GO thin film when the device is immersed in a liquid featuring higher ionic strength. For this reason, one can electrically track different washing cycles of the device operating in sweat by fixing $V_{G S}$ and $V_{D S}$ (Fig. 4d). Another crucial aspect is the device sensitivity. The rule of thumb in LGT sensing applications demands one to fix $V_{G S}$ at the maximum $g_{m}{ }^{[62]}$. Here, rGO-based CoG-LGTs showed the highest sensitivities around $1400 \mathrm{mVl}$, whereas the lowest values were observed close to the $I_{D S}^{\min }$ point (Fig. S17). An additional advantage of using permeable membranes is 


\section{WILEY-VCH}

the possibility to perform electromechanical assays, which is a nonviable practice by using traditional LGTs containing a PDMS reservoir and/or a TG electrode. This is a relevant issue as wearables are expected to conform in different shapes and curvatures. As shown in Fig. 4e and inset, the membrane-coated CoG-LGTs have been bent and simultaneously measured in sweat at three bending radii $(R)$ equal to $0 \mathrm{~cm}$ (i.e. flat), $0.75 \mathrm{~cm}$, and $1.15 \mathrm{~cm}$. I-V transfer characteristics exhibited only minor changes in the device response, thus providing unambiguous evidence for its robustness. Since no relevant electromechanical stresses and related failures have been observed, the LGT potentiometric sensitivity was evaluated at a severe bending condition, i.e., with $R=0.75 \mathrm{~cm}$ (Fig. 4f). Despite such a severe bending, the device potentiometric response remained virtually unchanged when compared to the flat condition (Fig. 3e), with $\Delta V_{G S}$ detectability down to $2 \mathrm{mV}$ with $\mathrm{S} / \mathrm{N}$ ratio equal to 63 and response times $<1 \mathrm{~s}$.

The benefit of coupling permeable membranes with LGTs can be further exploited to drive in or drain out solutions to and from the device without using traditional microfluidics. Aiming at the development of wearable LGTs, the need to electrically track a dynamic phenomenon such as perspiration is highly relevant ${ }^{[59-61]}$. As previously shown for static measurements, the permeable membrane has been proved to be an efficient approach for confining liquids. For driving the sweat in and out the device, we have conceived two strategies (Fig. 5). Layout I is envisaged to guide the sweat from a reservoir (e.g. the skin) towards a collector (viz. a fabric or another absorbent textile), with the device placed in between these two sites (Fig. 5a). For this purpose, a stripe of lateral flow (LF) paper is used to bridge the chip and the opposite sites. In other words, the layout I resembles a conventional microfluidic cell with a single straight channel endowed with an inlet (viz. reservoir) and an outlet (viz. collector). Layout II has been designed to outflow the sweat from the top of the device covered by a permeable membrane (Fig. 5b). Two collectors are placed sideways, and they are bridged by two LF paper stripes. Both approaches guarantee a continuous amount of 


\section{WILEY-VCH}

fresh sweat onto the chip, which is crucial for applications envisioning real-time

monitoring ${ }^{[55]}$. Within this context, we performed multiple injections of bi-distilled water and artificial sweat while recording the LGT time-dependent $I_{D S}$ at fixed potentials $\left(V_{G S}=-0.2 \mathrm{~V}\right.$ and $\left.V_{D S}=0.1 \mathrm{~V}\right)$. At such an experimental condition, the device $I_{D S}$ is expected to increase in the presence of water and to decrease in artificial sweat, as observed for device transfer curves (Fig. 4c). As exhibited in Fig. 5c,d, both layouts succeeded in transporting sweat and water in and out the device, respectively, while recording the device response in real-time. These experiments not only validate the innovation and effectiveness of our technology, but they also highlight the versatility of the devised approach for the development of wearable devices. As a result, these two layouts represent two opposite ways of application of wearable LGTs (Fig. 5e,f). On the one hand, layout I enables to place the electronic core outwards, thereby exploiting the PET safeguard that guarantees flexibility but not liquid permeation once in touch with the skin. On the other hand, layout II is faced towards the skin, thus making the electrical readout faster than in layout I as sweat will be directly carried onto the transducer. Both layouts, here named facing-up and facing-down wearable CoG LGTs, have pros and cons, yet they display an extreme versatility as major advantage. In fact, since each specific application will demand a customized solution, it is crucial to employ fabrication and assembly processes which are easily and fully adaptable.

\section{Conclusions}

We have fabricated a radically new flexible LGTs based on rGO displaying state-of-the-art electrical performance, for wearable electronics. The reported strategy combines for the first time: i) high-yield ( $\geq 99 \%$ ), scalable fabrication of LGTs onto PET substrates by laser ablation, ii) the use of a polycationic (viz. PDDA) adhesive primer for GO casting, and iii) the combination of a coplanar architecture with lateral-flow paper for real-time LGT operation in sweat. To the best of our knowledge, the reported approach boasts of peculiar features not 


\section{WILEY-VCH}

covered by the current rGO-based LGT technology. In particular, the in-situ GO electrochemical reduction in bi-distilled water made it possible to achieve a fully-green, lowcost and large-area spatially resolved device patterning. Finally, we have demonstrated an innovative and simple strategy based on lateral-flow paper and permeable membranes to confine and guide liquids, especially sweat, in and out the LGT active region. We have succeeded in monitoring LGTs in real-time while operating in artificial sweat in both dynamic and static modes, and also while subjecting the substrates to mechanical stress by changing the bending radii. These results open up novel technological avenues for applications in the field of flexible and wearables electronics, aiming at the development of sportswear or diagnostic point-of-care assays.

\section{Experimental Section}

Materials: Silicon (n-doped)/silicon dioxide $\left(\mathrm{Si} / \mathrm{SiO}_{2}\right)$ substrates (thermal oxide $90 \mathrm{~nm}$-thick, Fraunhofer), 4-inch wafer pyrex glass substrate (700 $\mu \mathrm{m}$ thick), polyethylene terephthalate (PET) substrates (160 um-thick), 3-(aminopropyl)triethoxysilane (APTES, Sigma Aldrich), poly(diallylmethylammonium chloride) solution (PDDA, Sigmal Aldrich), graphene oxide (GO, $4 \mathrm{mg} / \mathrm{mL}$, monolayer content $>$ 95\%, Graphenea), photoresin AZ1505 (Microchemicals), AZ726MIF developer (Microchemicals), hexamethyldisilazane (HMDS, Sigma Aldrich), polydimethylsiloxane (PDMS) elastomer resin and curing agent (Dow Corning), polyvinyl fluoride (PVDF) membranes (120 um-thick, 0.1 and $0.22 \mu \mathrm{m}$ pore size), polyether sulfone (PES) film (120 um-thick, $0.1 \mu \mathrm{m}$ pore size), Whatman ${ }^{\circledR}$ filter paper (180 $\mu$ m-thick, Sigma-Aldrich), CN 140 nitrocellulose membrane (130 $\mu$ m-thick, Unisart $\left.{ }^{\circledR}\right)$, lateral flow (LF) paper stripes (polyester-backed nitrocellulose, $200 \mu \mathrm{m}$-thick, mdi Membranes Technology), artificial sweat ISO-3160-2 (Reagents) containing $20 \mathrm{~g} / \mathrm{L} \mathrm{NaCl}$, $17.5 \mathrm{~g} / \mathrm{L} \mathrm{NH} \mathrm{N}_{4} \mathrm{Cl}, 1.5 \mathrm{~g} / \mathrm{L}$ lactic acid, $5 \mathrm{~g} / \mathrm{L}$ urea and $2.5 \mathrm{~g} / \mathrm{L}$ acetic acid, with $\mathrm{pH}$ adjusted to 4.7 with $\mathrm{NaOH}$. 


\section{WILEY-VCH}

Fabrication of LGTs test pattern: LGTs have been fabricated on $15 \mathrm{~cm} \times 15 \mathrm{~cm} \mathrm{Si} / \mathrm{SiO}_{2}$, glass, and PET test patterns. $\mathrm{Si} / \mathrm{SiO}_{2}$ chips containing from one to four pairs of $\mathrm{Au} / \mathrm{Cr}(40 / 20 \mathrm{~nm}-$ thick) interdigitated electrodes (IDEs) were fabricated by maskless photolithography and liftoff. $\mathrm{Si} / \mathrm{SiO}_{2}$ test patterns bearing a coplanar gate electrode $(\mathrm{CoG})$ were manufactured by the same process. Glass test patterns were manufactured by screen printing technology having from one to four $\mathrm{Au} / \mathrm{Ti}$ IDEs (90/10 nm-thick). Finally, PET test patterns were fabricated by laser ablation at the laboratories of Metrohm DropSens (Oviedo, Spain). For this purpose, PET foils were sputter-coated with a $20 \mathrm{~nm}$-thick Au layer and further ablated by an Nd:YAG laser (532 nm and $1064 \mathrm{~nm}$ wavelengths, adjustable power) in an automated laser scribing station (Rofin, Germany). The IDEs dimensions, such as channel width $(W)$ and length $(L)$, and disposition onto the respective substrates are dependent on the fabrication technology. Such details are provided in the Supporting Information (SI) as Fig. S1.

Casting of the graphene oxide film: LGTs were fabricated based on a commercial GO water dispersion diluted 1:10 featuring a final concentration of $0.4 \mathrm{mg} / \mathrm{mL}$. Prior to being used, the GO suspension was sonicated for $1 \mathrm{~min}$ for a better homogeneity. $\mathrm{Si} / \mathrm{SiO}_{2}$ and glass testbeds were copiously rinsed in acetone and isopropanol for cleaning, then further activated for 20 min by UV/ozone treatment (NovaScan, Digital UV/Ozone System). Afterwards, the substrates were immersed in APTES solution in toluene $(1 \% \mathrm{v} / \mathrm{v})$ for $30 \mathrm{~min}$, rinsed, and subsequently immersed in GO for 3h. PET substrates were cleaned in isopropanol and no UV/Ozone activation was employed. The PET substrates were immersed for $15 \mathrm{~min}$ in a PDDA aqueous solution ( $1 \% \mathrm{w} / \mathrm{w})$ containing $0.5 \mathrm{M}$ of $\mathrm{NaCl}$, rinsed thoroughly, and immersed in GO for 3h. After the GO deposition, all coated substrates were copiously rinsed in bi-distilled water and blown dry in $\mathrm{N}_{2}$. 


\section{WILEY-VCH}

GO electrochemical reduction: Here, we employed the on-site electrochemical reduction of GO as part of the device fabrication steps to reduce and pattern the LGT channel region. The GO electrochemical reduction was carried out by cyclic voltammetry $(\mathrm{CV})$ by using a Metrohm Autolab PGSTAT204 potentiostat/galvanostat. For this purpose, the test pattern source (S) and drain (D) IDEs have been short-circuited to act as the working electrode (WE) in the electrochemical setup. An external polycrystalline Au plate (area $c a .0 .031 \mathrm{~cm}^{2}$ ) was employed as a counter (CE)/reference (RE) single electrode. Few (1-4) voltammetric cycles are sufficient to achieve the GO reduction. The applied potential has been swept from $0 \mathrm{~V}$ to $2.8 \mathrm{~V}$ at a scan rate of $100 \mathrm{mV} / \mathrm{s}$ in bi-distilled water.

Prior to the reduction cycle, the Au plate was cleaned in $\mathrm{H}_{2} \mathrm{SO}_{4}(95-97 \%$, Sigma-Aldrich) and by UV/ozone exposure for 20 min each.

Spectroscopic and morphological characterization: GO and rGO films have been characterized by X-ray photoelectron spectroscopy (XPS), Raman spectroscopy, Atomic Force Microscopy (AFM) and Scanning Electron Microscopy (SEM). XPS analyses were carried out by using a Thermo Scientific K-Alpha X-ray photoelectron spectrometer equipped with an aluminum X-ray source (energy $1.4866 \mathrm{keV}$ ). The spectra have been recorded at a pressure of $10^{-8}$ mbar in the main chamber. The X-ray spot size was settled at $400 \mu \mathrm{m}$. Survey spectra were recorded as a result of 10 scans with a pass energy of $200.00 \mathrm{eV}$ and a step size of $1 \mathrm{eV}$; high-resolution spectra were an average of 10 scans with a pass energy of $50.00 \mathrm{eV}$ and a step size of $0.1 \mathrm{eV}$.

Raman measurements were conducted with a Renishaw InVia Reflex system. The spectrograph used a high-resolution grating (2400 grooves $\mathrm{cm}^{-1}$ ) with additional band-pass filter optics, a confocal microscope, and a 2D-CCD camera. Laser excitation was carried out at $532 \mathrm{~nm}$ with a $100 \times$ objective (N.A. 0.85 ), $0.33 \mathrm{~mW}$ of maximum power, and $10 \mathrm{~s}$ 


\section{WILEY-VCH}

acquisition time. Raman images were obtained using a Raman point-mapping method with a $100 \times$ objective (N.A. 0.85 ), which provided a spatial resolution of about $0.46 \mu \mathrm{m}^{2}$. It creates a spectral image by measuring the Raman spectrum of each pixel of the image, one at a time. The Raman images of each well were decoded using the characteristic peak intensities of the GO using WiRE software V 4.2 (Renishaw, U.K.).

AFM characterization was carried out in a Bruker Dimension Icon microscope, in intermittent contact mode using a TESPA-V2 silicon tip with a $42 \mathrm{~N} / \mathrm{m}$ force constant. SEM imaging was conducted using an FEI Quanta 250 FEG instrument operated in high vacuum, with accelerating voltages of $2-5 \mathrm{kV}$ for the incident beam.

Electrical measurements: LGTs have been electrically characterized by DC and AC measurements. The device operation was monitored by measuring their transfer $\left(I_{D S}-V_{G S}\right)$ and output $\left(I_{D S}-V_{D S}\right)$ characteristics. The LGT transfer curves were obtained by sweeping the gate electrode potential $\left(V_{G S}\right)$ from $-0.4 \mathrm{~V}$ to $+0.8 \mathrm{~V}$, unless otherwise stated, at a rate of $20 \mathrm{mV} / \mathrm{s}$ and at a constant source-drain bias $\left(V_{D S}\right)$ of $100 \mathrm{mV}$. The devices were subjected to 3-5 transfers scans until a stable response could be attained. The LGT output characteristics were acquired sweeping $V_{D S}$ from $0 \mathrm{~V}$ to $100 \mathrm{mV}$ at positive and negative $V_{G S}$. In both measurements, the device drain-source $\left(I_{D S}\right)$ and gate-source $\left(I_{G S}\right)$ currents were monitored. The LGT potentiometric sensitivity and response time was evaluated at a constant $V_{D S}$ for different gate voltage modulation $\left(\Delta V_{G S}\right)$ by monitoring the device $I_{D S}$ as a function of time. The acquisition interval for each current point was $10 \mathrm{~ms}$. All DC electrical measurements were carried out in a probe station, in ambient conditions, using a Keithley 2626A SourceMeter unit.

AC measurements were performed by means of electrochemical impedance spectroscopy (EIS) employing a Metrohm Autolab PGSTAT204 potentiostat/galvanostat. The CoG electrode was used as the WE and the short-circuited $\mathrm{S}$ and $\mathrm{D}$ terminals as the CE/RE 


\section{WILEY-VCH}

terminal. The impedance spectrum was recorded in the frequency range of $10^{-1}$ to $10^{5} \mathrm{~Hz}$, swept from high to low frequencies, with sine-wave voltage signal amplitude of $100 \mathrm{mV}$ (root-mean-square, rms). The device complex impedance $\left(Z^{*}\right)$ was converted into capacitance $\left(C_{p}\right)$ using a simple parallel resistor-capacitor (RC) circuit.

\section{Supporting Information}

Supporting Information is available from the Wiley Online Library or from the author.

\section{Acknowledgements}

We acknowledge funding from the European Commission through the Graphene Flagship Core 2 project (GA-785219), the AMI project funded by the ERA-NET EuroNanoMed III programme, the European Union and the Agence Nationale de la Recherche (ANR) GAANR-17-ENM3-0001-01, the Marie Sklodowska-Curie projects ITN project BORGES (GA813863), the Agence Nationale de la Recherche through the Labex projects CSC (ANR-10LABX-0026 CSC) and NIE (ANR-11-LABX-0058 NIE) within the Investissement d'Avenir program (ANR-10-120 IDEX-0002-02), and the International Center for Frontier Research in Chemistry (icFRC). We aknowledge Dr. T. Cramer (University of Bologna/Italy) for support with accurate potentiometric measurements.

Received: ((will be filled in by the editorial staff))

Revised: ((will be filled in by the editorial staff)) Published online: ((will be filled in by the editorial staff))

\section{References}

[1] K. S. Novoselov, A. K. Geim, S. V. Morozov, D. Jiang, Y. Zhang, S. V. Dubonos, I. V. Grigorieva, A. A. Firsov, Science 2004, 306, 666.

[2] A. C. Ferrari, F. Bonaccorso, V. Fal'ko, K. S. Novoselov, S. Roche, P. Bøggild, S.

Borini, F. H. L. Koppens, V. Palermo, N. Pugno, J. A. Garrido, R. Sordan, A. Bianco, L.

Ballerini, M. Prato, E. Lidorikis, J. Kivioja, C. Marinelli, T. Ryhänen, A. Morpurgo, J. N.

Coleman, V. Nicolosi, L. Colombo, A. Fert, M. Garcia-Hernandez, A. Bachtold, G. F.

Schneider, F. Guinea, C. Dekker, M. Barbone, Z. Sun, C. Galiotis, A. N. Grigorenko, G.

Konstantatos, A. Kis, M. Katsnelson, L. Vandersypen, A. Loiseau, V. Morandi, D. Neumaier,

E. Treossi, V. Pellegrini, M. Polini, A. Tredicucci, G. M. Williams, B. Hee Hong, J. H. Ahn, J.

Min Kim, H. Zirath, B. J. Van Wees, H. Van Der Zant, L. Occhipinti, A. Di Matteo, I. A. 


\section{WILEY-VCH}

Kinloch, T. Seyller, E. Quesnel, X. Feng, K. Teo, N. Rupesinghe, P. Hakonen, S. R. T. Neil,

Q. Tannock, T. Löfwander, J. Kinaret, Nanoscale 2015, 7, 4598.

[3] M. J. Allen, V. C. Tung, R. B. Kaner, Chem. Rev. 2010, 110, 132.

[4] N. O. Weiss, H. Zhou, L. Liao, Y. Liu, S. Jiang, Y. Huang, X. Duan, Adv. Mater. 2012, 24,5782 .

[5] S. Kabiri Ameri, R. Ho, H. Jang, L. Tao, Y. Wang, L. Wang, D. M. Schnyer, D.

Akinwande, N. Lu, ACS Nano 2017, 11, 7634.

[6] H. Lee, T. K. Choi, Y. B. Lee, H. R. Cho, R. Ghaffari, L. Wang, H. J. Choi, T. D.

Chung, N. Lu, T. Hyeon, S. H. Choi, D. H. Kim, Nat. Nanotechnol. 2016, 11, 566.

[7] J. Kim, M. Kim, M. S. Lee, K. Kim, S. Ji, Y. T. Kim, J. Park, K. Na, K. H. Bae, H. K. Kim, F. Bien, C. Y. Lee, J. U. Park, Nat. Commun. 2017, 8, 1.

[8] D. Chen, H. Feng, J. Li, Chem. Rev. 2012, 112, 6027.

[9] S. Stankovich, D. A. Dikin, G. H. B. Dommett, K. M. Kohlhaas, E. J. Zimney, E. A.

Stach, R. D. Piner, S. B. T. Nguyen, R. S. Ruoff, Nature 2006, 442, 282.

[10] C. Gómez-Navarro, R. T. Weitz, A. M. Bittner, M. Scolari, A. Mews, M. Burghard, K. Kern, Nano Lett. 2007, 7, 3499.

[11] H. C. Schniepp, J. L. Li, M. J. McAllister, H. Sai, M. Herrera-Alonson, D. H.

Adamson, R. K. Prud'homme, R. Car, D. A. Seville, I. A. Aksay, J. Phys. Chem. B 2006, 110, 8535.

[12] X. Wang, L. Zhi, K. Müllen, Nano Lett. 2008, 8, 323.

[13] G. Eda, G. Fanchini, M. Chhowalla, Nat. Nanotechnol. 2008, 3, 270.

[14] S. Pei, H. M. Cheng, Carbon 2012, 50, 3210.

[15] C. Anichini, W. Czepa, D. Pakulski, A. Aliprandi, A. Ciesielski, P. Samorì, Chem. Soc. Rev. 2018, 47, 4860 .

[16] R. Stine, J. T. Robinson, P. E. Sheehan, C. R. Tamanaha, Adv. Mater. 2010, 22, 5297. 


\section{WILEY-VCH}

[17] M. Larisika, C. Kotlowski, C. Steininger, R. Mastrogiacomo, P. Pelosi, S. Schütz, S. F.

Peteu, C. Kleber, C. Reiner-Rozman, C. Nowak, W. Knoll, Angew. Chemie - Int. Ed. 2015, 54, 13245.

[18] E. Piccinini, C. Bliem, C. Reiner-Rozman, F. Battaglini, O. Azzaroni, W. Knoll, Biosens. Bioelectron. 2017, 92, 661.

[19] H. G. Sudibya, Q. He, H. Zhang, P. Chen, ACS Nano 2011, 5, 1990.

[20] C. Reiner-Rozman, M. Larisika, C. Nowak, W. Knoll, Biosens. Bioelectron. 2015, 70, 21.

[21] I. Y. Sohn, D. J. Kim, J. H. Jung, O. J. Yoon, T. Nguyen Thanh, T. Tran Quang, N. E. Lee, Biosens. Bioelectron. 2013, 45, 70.

[22] Q. He, H. G. Sudibya, Z. Yin, S. Wu, H. Li, F. Boey, W. Huang, P. Chen, H. Zhang, ACS Nano 2010, 4, 3201.

[23] Q. He, S. Wu, S. Gao, X. Cao, Z. Yin, H. Li, P. Chen, H. Zhang, ACS Nano 2011, 5, 5038.

[24] T. Cramer, A. Campana, F. Leonardi, S. Casalini, A. Kyndiah, M. Murgia, F. Biscarini, J. Mater. Chem. B 2013, 1, 3728.

[25] S. Casalini, A. C. Dumitru, F. Leonardi, C. A. Bortolotti, E. T. Herruzo, A. Campana, R. F. De Oliveira, T. Cramer, R. Garcia, F. Biscarini, ACS Nano 2015, 9, 5051.

[26] S. J. Park, O. S. Kwon, S. H. Lee, H. S. Song, T. H. Park, J. Jang, Nano Lett. 2012, 12, 5082.

[27] H. Li, J. Zhang, X. Zhou, G. Lu, Z. Yin, G. Li, T. Wu, F. Boey, S. S. Venkatraman, H. Zhang, Langmuir 2010, 26, 5603.

[28] M. C. Kim, G. S. Hwang, R. S. Ruoff, J. Chem. Phys. 2009, 131, 1.

[29] N. A. Kotov, I. Dekani, J. H. Fendler, Adv. Mater. 1996, 6, 637.

[30] H. A. Becerril, J. Mao, R. M. Stoltenberg, Z. Bao, Y. Chen, ACS Nano 2008, 2, 463. 


\section{WILEY-VCH}

[31] Y. L. Zhang, L. Guo, H. Xia, Q. D. Chen, J. Feng, H. B. Sun, Adv. Opt. Mater. 2014, 2, 10.

[32] D. Voiry, R. Fullon, C. Lee, H. Y. Jeong, H. S. Shin, M. Chhowalla, Science 2016, $353,1430$.

[33] C. N. Hernández, M. B. G. García, D. H. Santos, M. A. Heras, A. Colina, P. FanjulBolado, Electrochem. Commun. 2016, 64, 65.

[34] Y. Shao, J. Wang, M. Engelhard, C. Wang, Y. Lin, J. Mater. Chem. 2010, 20, 743.

[35] S. J. An, Y. Zhu, S. H. Lee, M. D. Stoller, T. Emilsson, S. Park, A. Velamakanni, J. An, R. S. Ruoff, J. Phys. Chem. Lett. 2010, 1, 1259.

[36] G. K. Ramesha, S. Sampath, J. Phys. Chem. C 2009, 113, 7985.

[37] M. Zhou, Y. Wang, Y. Zhai, J. Zhai, W. Ren, F. Wang, S. Dong, Chem. - A Eur. J. 2009, 15, 6116.

[38] K. Balasubramanian, M. Friedrich, C. Jiang, Y. Fan, A. Mews, M. Burghard, K. Kern, Adv. Mater. 2003, 15, 1515.

[39] J.M. Mativetsky, A. Liscio, E. Treossi, E. Orgiu, A. Zanelli, P. Samorì, V. Palermo, J. Am. Chem. Soc. 2011, 133, 14320.

[40] J. M. Mativetsky, E. Treossi, E. Orgiu, M. Melucci, G. P. Veronese, P. Samorì, V. Palermo, J. Am. Chem. Soc. 2010, 132, 14130.

[41] A. C. Ferrari, D. M. Basko, Nat. Nanotechnol. 2013, 8, 235.

[42] F. J. Ibañez, F. P. Zamborini, Small 2012, 8, 174.

[43] B. Raguse, E. Chow, C. S. Barton, L. Wieczorek, Anal. Chem. 2007, 79, 7333.

[44] Z. Wei, D. E. Barlow, P. E. Sheehan, Nano Lett. 2008, 8, 3141.

[45] R. F. De Oliveira, S. Casalini, T. Cramer, F. Leonardi, M. Ferreira, V. Vinciguerra, V. Casuscelli, N. Alves, M. Murgia, L. Occhipinti, F. Biscarini, Flex. Print. Electron. 2016, 1, 1. [46] Y. Lvov, G. Decher, M. Mohwald, Langmuir 1993, 9, 481. 


\section{WILEY-VCH}

[47] L. Kergoat, L. Herlogsson, D. Braga, B. Piro, M.-C. C. Pham, X. Crispin, M. Berggren, G. Horowitz, Adv. Mater. 2010, 22, 2565.

[48] R. F. De Oliveira, L. Merces, T. P. Vello, C. C. Bof Bufon, Org. Electron. 2016, 31, 217.

[49] H. Toss, C. Suspène, B. Piro, A. Yassar, X. Crispin, L. Kergoat, M. C. Pham, M. Berggren, Org. Electron. 2014, 15, 2420.

[50] C. Cheng, G. Jiang, G. P. Simon, J. Z. Liu, D. Li, Nat. Nanotechnol. 2018, 13, 685.

[51] F. Leonardi, S. Casalini, Q. Zhang, S. Galindo, D. Gutiérrez, M. Mas-torrent, Adv. Mater. 2016, 28, 10311-10316.

[52] T. Cramer, A. Kyndiah, M. Murgia, F. Leonardi, S. Casalini, F. Biscarini, Appl. Phys. Lett. 2012, 100, 143302.

[53] D. Khodagholy, T. Doublet, P. Quilichini, M. Gurfinkel, P. Leleux, A. Ghestem, E. Ismailova, T. Hervé, S. Sanaur, C. Bernard, G. G. Malliaras, Nat. Commun. 2013, 4, 1575.

[54] D. Khodagholy, J. N. Gelinas, T. Thesen, W. Doyle, O. Devinsky, G. G. Malliaras, G. Buzsáki, Nat. Neurosci. 2015, 18, 310.

[55] M. Bariya, H. Y. Y. Nyein, A. Javey, Nat. Electron. 2018, 1, 160.

[56] D. B. Speedy, T. D. Noakes, C. Schneider, Emerg. Med. Australas. 2001, 13, 17.

[57] J. Heikenfeld, Nature 2016, 529, 475.

[58] Z. Sonner, E. Wilder, J. Heikenfeld, G. Kasting, F. Beyette, D. Swaile, F. Sherman, J. Joyce, J. Hagen, N. Kelley-Loughnane, R. Naik, Biomicrofluidics 2015, 9, 031301.

[59] S. Anastasova, B. Crewther, P. Bembnowicz, V. Curto, H. M. Ip, B. Rosa, G. Z. Yang, Biosens. Bioelectron. 2017, 93, 139.

[60] W. Gao, S. Emaminejad, H. Y. Y. Nyein, S. Challa, K. Chen, A. Peck, H. M. Fahad, H. Ota, H. Shiraki, D. Kiriya, D. H. Lien, G. A. Brooks, R. W. Davis, A. Javey, Nature 2016, $529,509$. 


\section{WILEY-VCH}

[61] B. Schazmann, D. Morris, C. Slater, S. Beirne, C. Fay, R. Reuveny, N. Moyna, D.

Diamond, Anal. Methods 2010, 2, 342.

[62] D. Khodagholy, J. Rivnay, M. Sessolo, M. Gurfinkel, P. Leleux, L. H. Jimison, E.

Stavrinidou, T. Herve, S. Sanaur, R. M. Owens, G. G. Malliaras, Nat. Commun. 2013, 4, 2133. 


\section{WILEY-VCH}
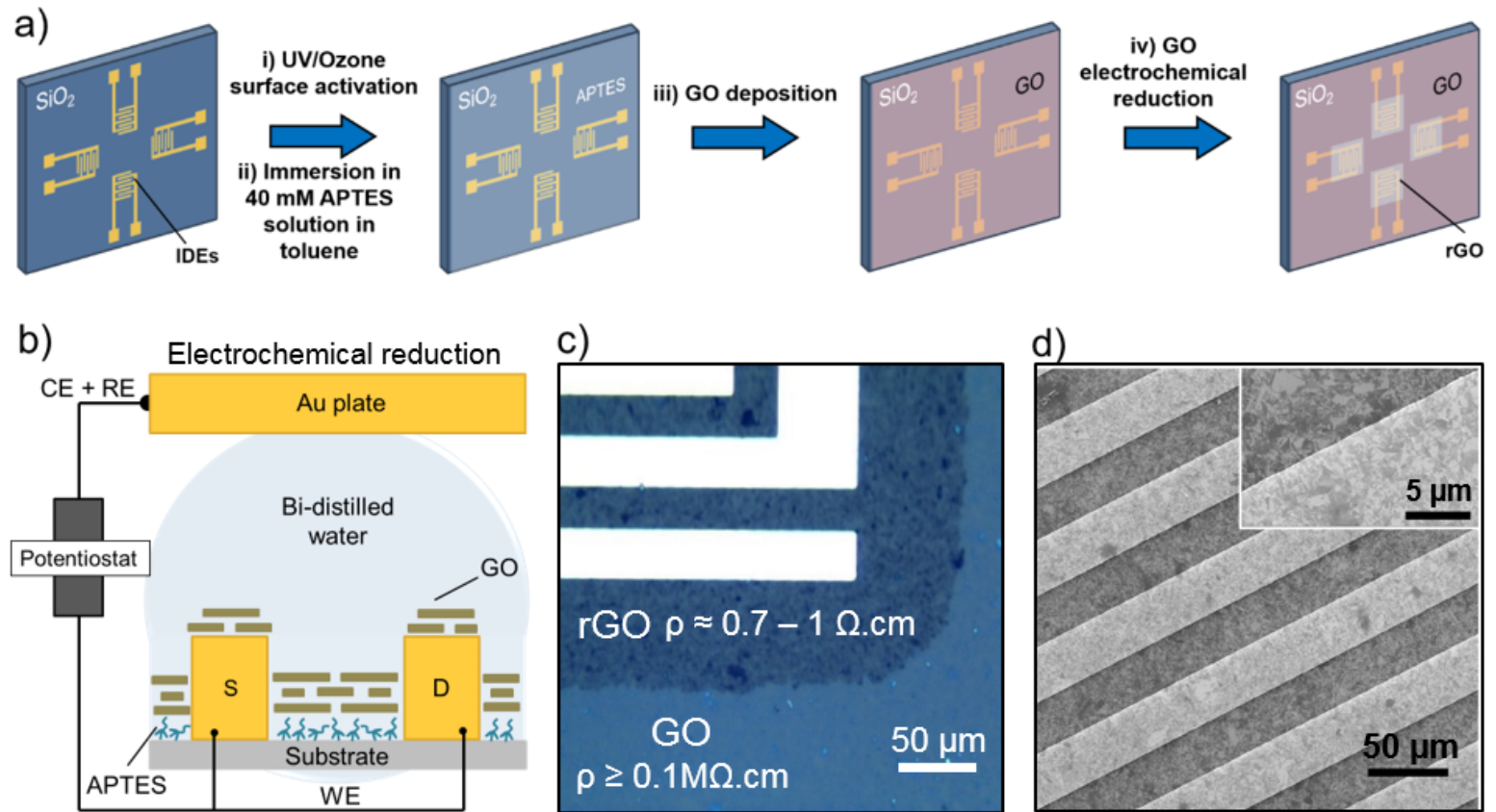

c)
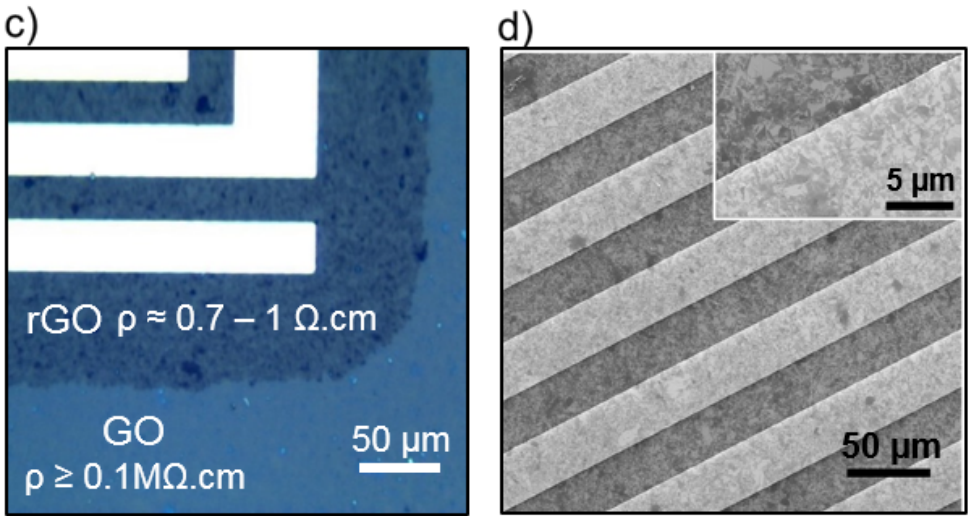

e)

f)

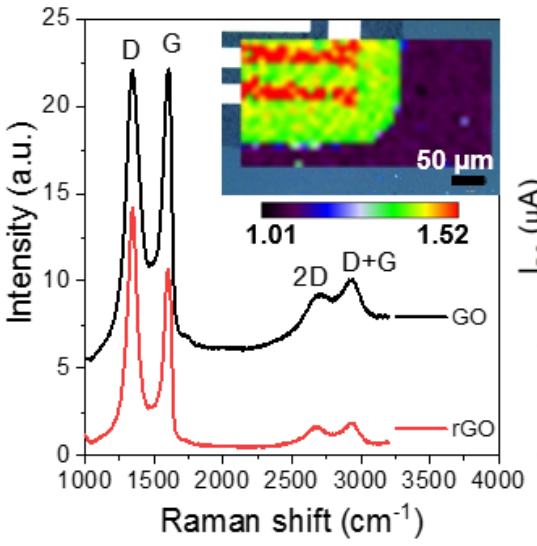

g)

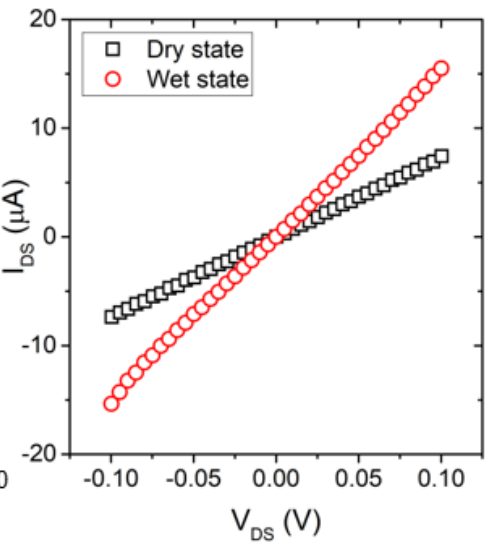

Figure 1. a) Fabrication protocol of rGO devices onto $\mathrm{Si} / \mathrm{SiO}_{2}$ test patterns. b) Sketch of the electrochemical setup for in-situ GO electrochemical reduction. c) Optical image of the $\mathrm{GO} / \mathrm{rGO}$ film regions and their respective electrical resistivities. d) SEM image of the rGO film on IDEs (inset for details). e) AFM image of the GO film morphology onto $\mathrm{Si} / \mathrm{SiO}_{2}$. f) Raman spectra of GO (black) and rGO (red) - vertical offset for clarity. Inset: Raman mapping of coated IDEs considering the measured D/G band intensity ratio. g) I-V plot for a two-terminal rGO device in dry state (empty black squares) and in bi-distilled water (empty red circles). 


\section{WILEY-VCH}

a)
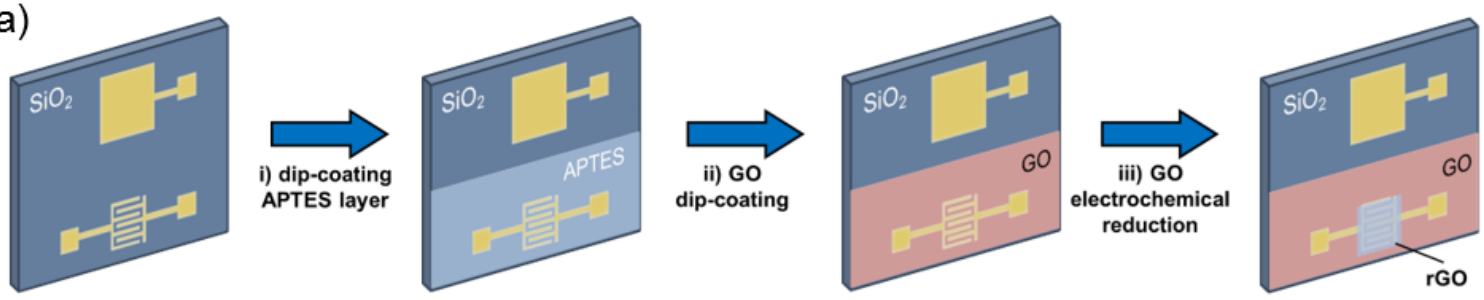

Coplanar gate

b)

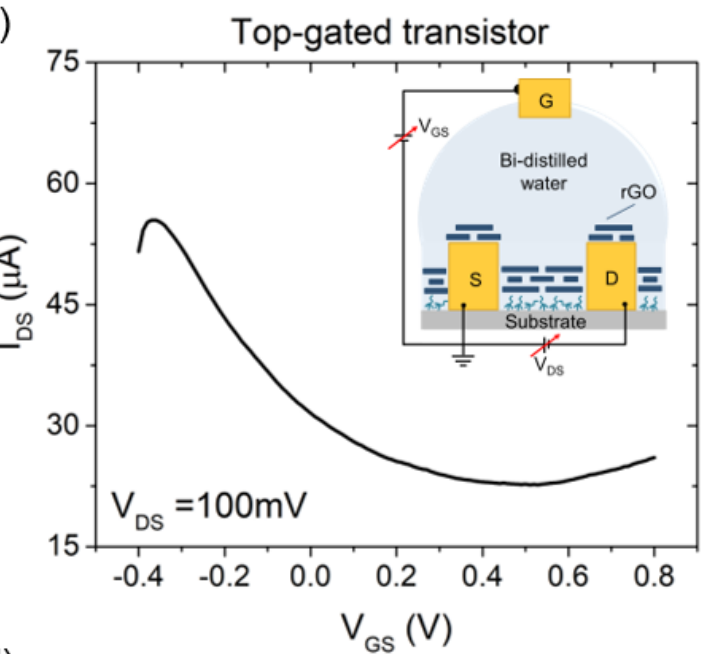

c)

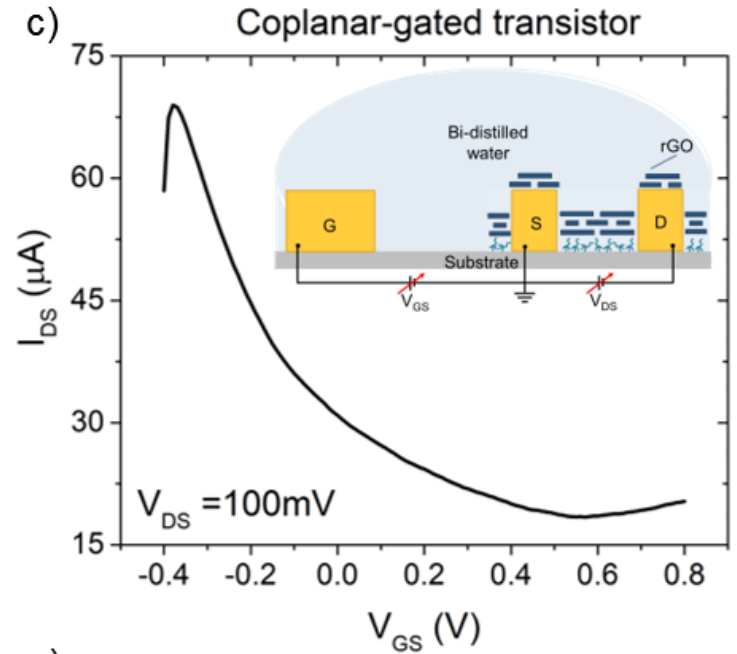

d)
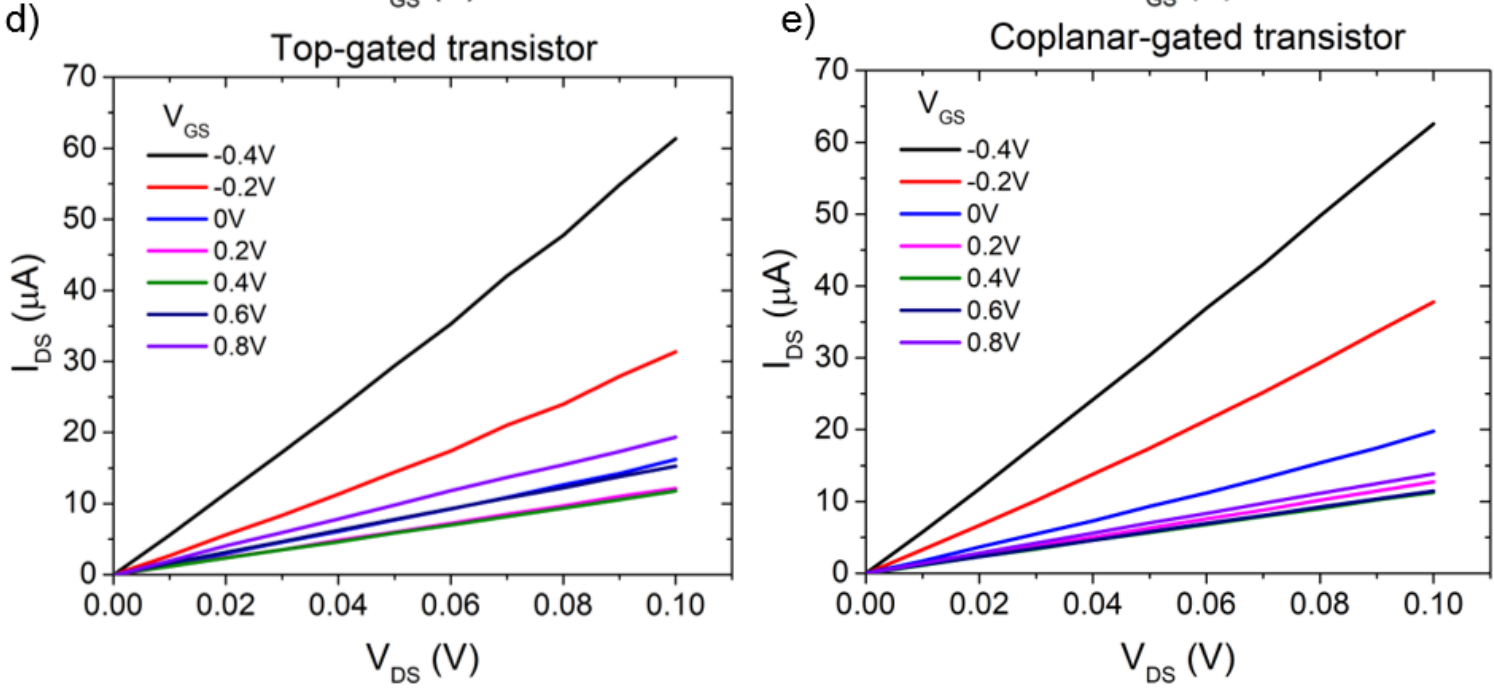

Figure 2. a) Partial dipping approach for GO patterning onto IDEs in a $\mathrm{CoG}$ device architecture. b,c) I-V transfer and $\mathbf{d}, \mathbf{e})$ output characteristics of $\mathrm{Si} / \mathrm{SiO}_{2}$ rGO TG-LGTs and CoG-LGTs, respectively. The insets in b) and c) illustrate the two device architectures and electrical connections. For TG-LGTs, an Au wire was employed as the gate electrode. 


\section{WILEY-VCH}

a)
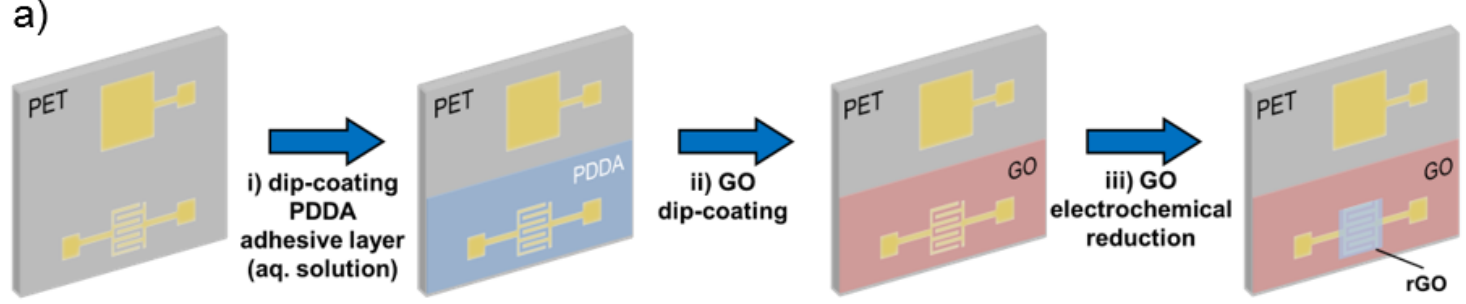

b)

c)
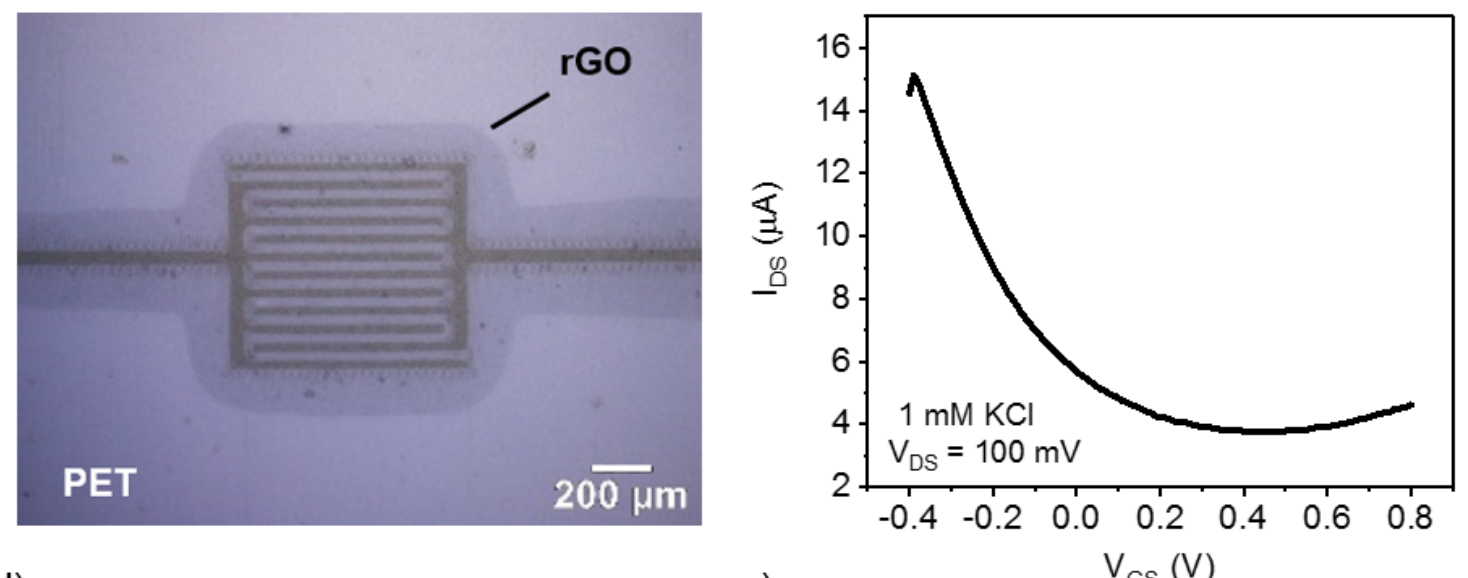

d)

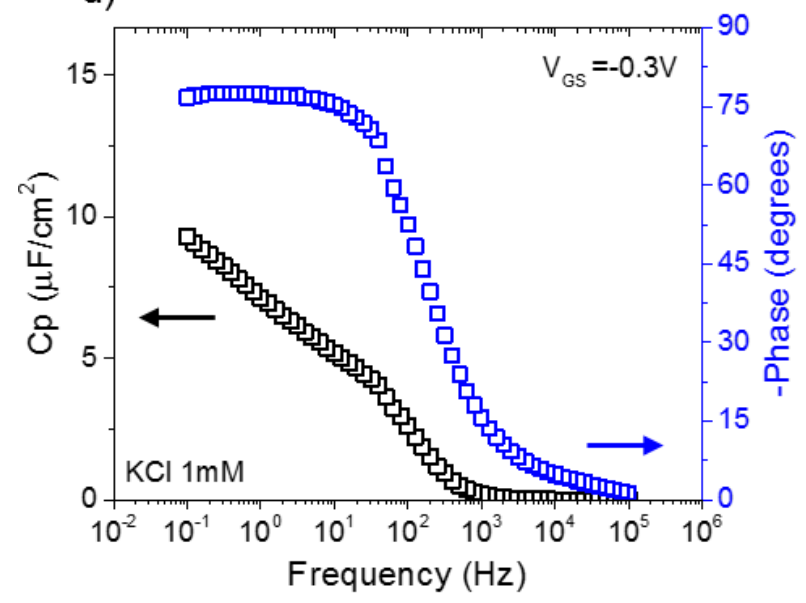

e)

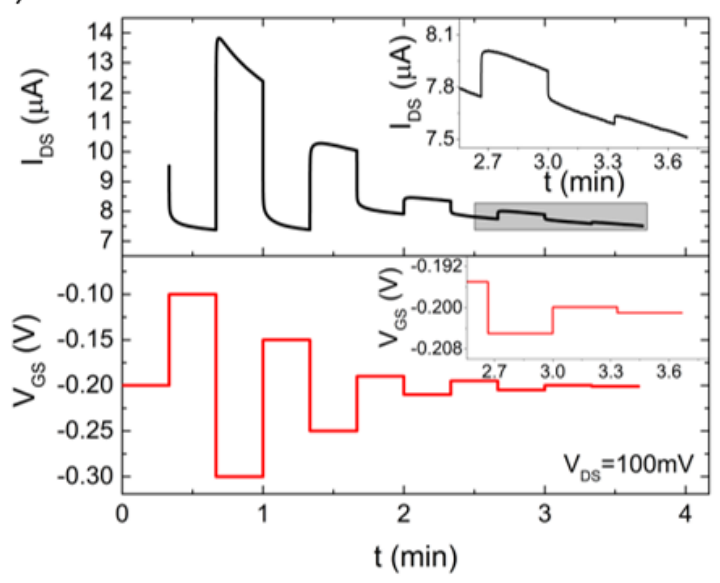

Figure 3 a) Partial test pattern dipping for GO assembly onto PET test patterning using a PDDA adhesive layer. b) Optical image (dark field) of rGO onto the PET IDEs. c) I-V transfer characteristics of PET/PDDA/rGO CoG LGTs in $1 \mathrm{mM} \mathrm{KCl}$. d) Capacitance $(C p)$ and impedance phase vs. frequency plot extracted by EIS of CoG-LGT. e) IDS vs. time featuring the potentiometric sensitivity of the rGO CoG-LGT. The inset shows details of the gate potential and current response on highlighted curve region. 
WILEY-VCH

a)

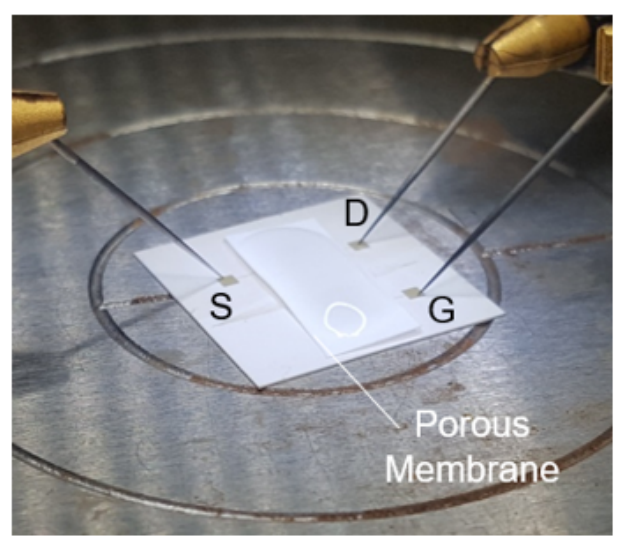

c)
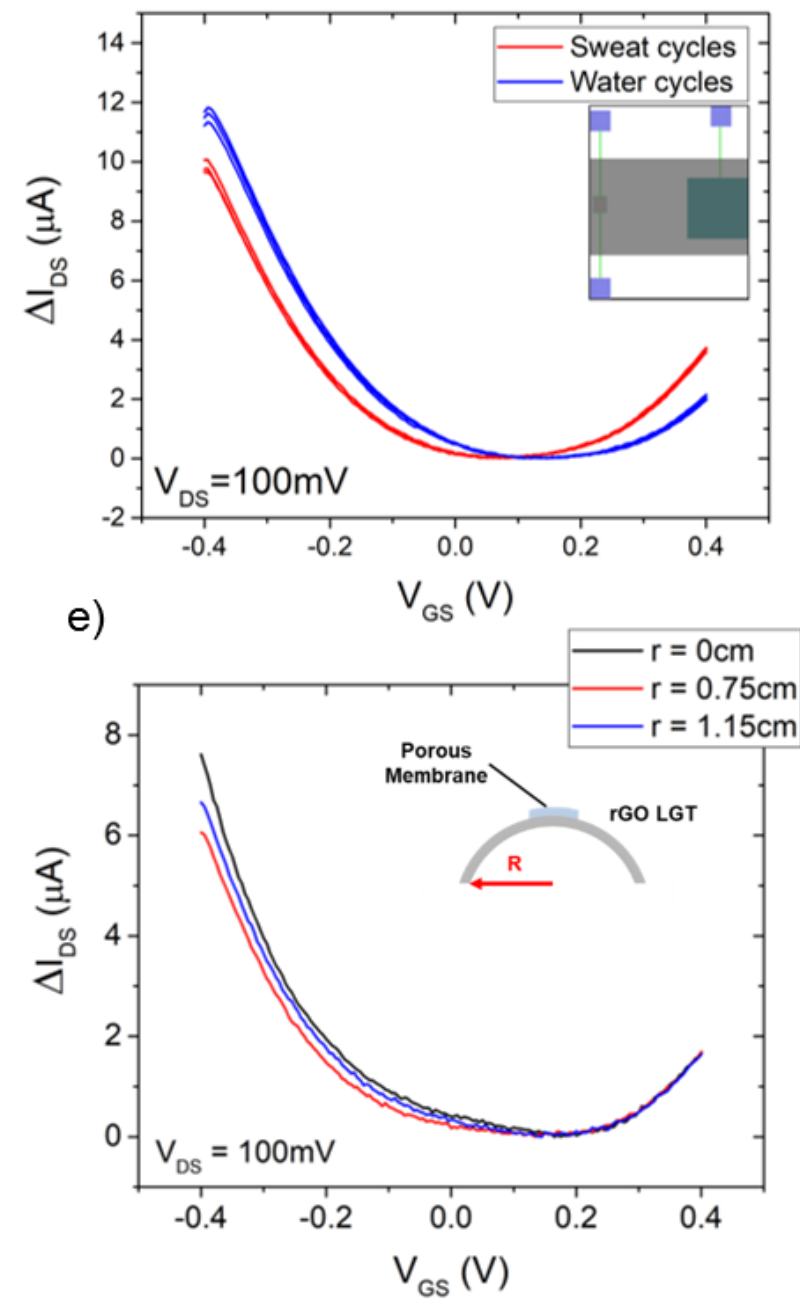

b)

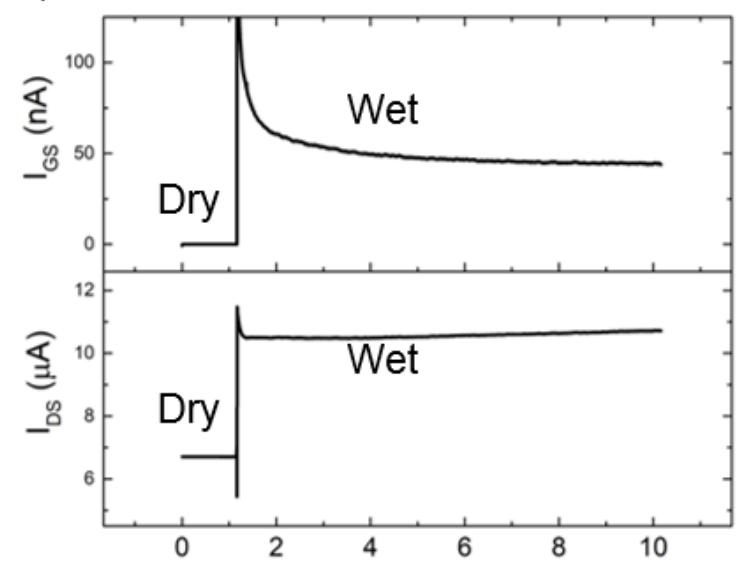

d)

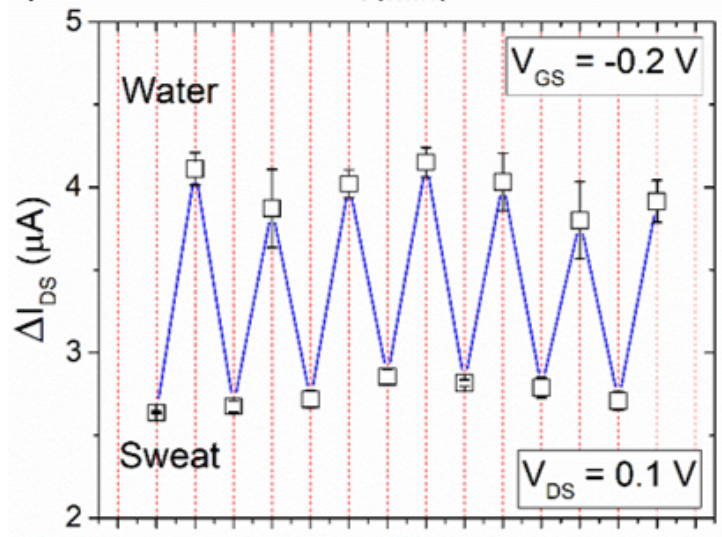

f)

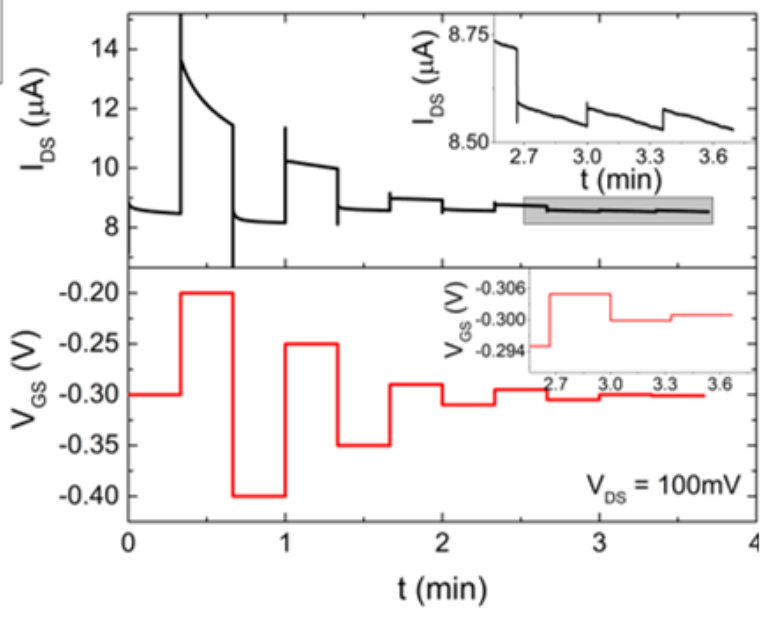

Figure 4 a) Picture of a PET rGO CoG-LGT covered by a strip of permeable membrane for sweat confinement. b) Membrane-covered CoG-LGT response ( $I_{D S}$ and $\left.I_{G S}\right)$ as a function of time during the device wetting process. c) I-V transfer characteristics ( 3 cycles) in bi-distilled water (blue line) and in artificial sweat (red line). d) $\mathrm{I}_{\mathrm{DS}}$ changes corresponding to sweat (lower points) and bi-distilled water (upper points) exposure cycles at $V_{D S}=100 \mathrm{mV}$ and $V_{G S}=-200 \mathrm{mV}$. e) I-V transfer characteristics in sweat for curved rGO CoG-LGTs on PET. Inset: bending schematics. f) Potentiometric stimulus and response of PET devices in sweat curved with bending radius of $0.75 \mathrm{~cm}$. The inset shows details of the $I_{D S}$ and $V_{G S}$ at the highlighted region. 

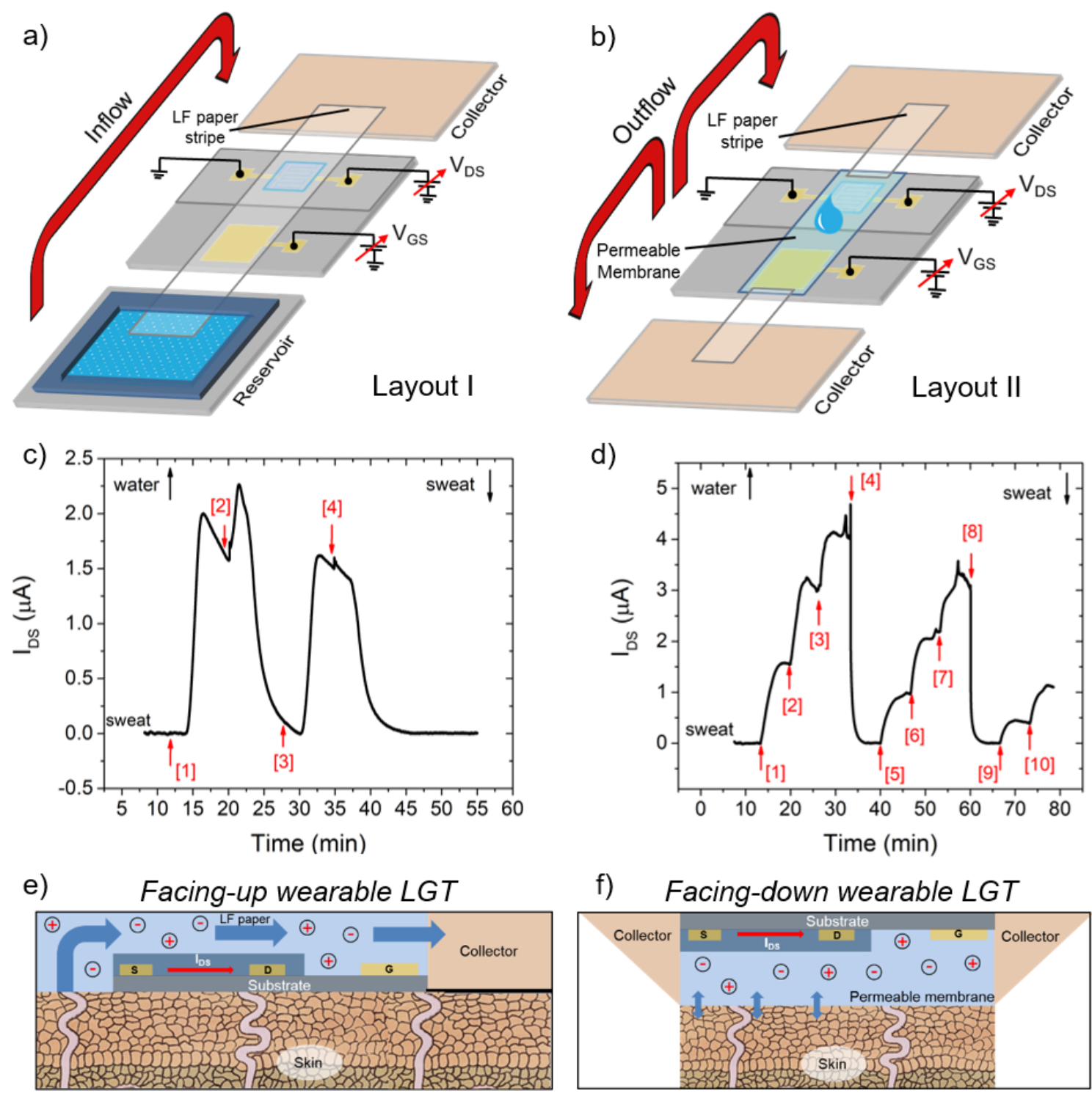

Figure 5 Fluid transportation in LGTs based on lateral-flow strategy. Schematics of the a) layout I and b) layout II and their respective real-time response (c,d) during multiple injections of sweat and bi-distilled water. Upward and downward arrows stand for water and sweat injections, respectively. (e,f) Envisioned applications of the two layouts (facing-up and facing-down LGTs) for wearable applications. 